\title{
BOUNDS FOR VOLUMES OF SUB-LEVEL SETS OF POLYNOMIALS AND APPLICATIONS
}

\author{
Ta Le Loi ${ }^{\mathrm{a}}$, Pham Minh Quy ${ }^{\mathrm{a}^{*}}$
}

${ }^{a}$ The Faculty of Mathematics and Computer Science, Dalat University, Lam Dong, Vietnam ${ }^{*}$ Corresponding author: Email: p.minhquydl@gmail.com

\author{
Article history \\ Received: March 25 $5^{\text {th }}, 2021$ \\ Received in revised form: May $14^{\text {th }}, 2021 \mid$ Accepted: May $19^{\text {th }}, 2021$ \\ Available online: January $27^{\text {th }}, 2022$
}

\begin{abstract}
In this paper, we present some explicit exponents in the estimates for the volumes of sub-level sets of polynomials on bounded sets and applications to the decay of oscillatory integrals and the convergence of singular integrals.
\end{abstract}

Keywords: Oscillatory integral; Polynomial; Singular integral; Sub-level set.

DOI: http://dx.doi.org/10.37569/DalatUniversity.12.2.875(2022)

Article type: (peer-reviewed) Full-length research article

Copyright $(2022$ The author(s)

Licensing: This article is licensed under a CC BY-NC 4.0 


\section{INTRODUCTION}

Let $f$ be a real function on $\mathbb{R}^{n}$ and $A$ be a subset of $\mathbb{R}^{n}$. We will denote by

$$
K_{A, f, t}=\{x \in A:|f(x)| \leq t\}
$$

the sub-level set of $f$ with $t \in \mathbb{R}_{\geq 0}$. We are interested in the estimates for the volumes of these sub-level sets:

$$
V(t)=\operatorname{Vol}\left(K_{A, f, t}\right), \text { when } t \rightarrow 0 .
$$

This problem has been considered by many mathematicians and has a close relation to the estimation of the decay of the oscillatory integral with phase $f$,

$$
I(\lambda)=\left|\int_{A} e^{i \lambda f(x)} d x\right|, \text { when } \lambda \rightarrow \infty
$$

and the convergence of the singular integral $\int_{A}|f(x)|^{-\gamma} d x$. These objects are central in many fields of physics and mathematics, including harmonis analysis and singularity theory. We refer to some of them (Arnold et al., 1988; Carbery et al., 1999; Cluckers \& Miller, 2016; Nguyen et al., 2018; Phong et al., 1999; Rogers, 2005; Stein, 1993; Ta, 2021).

In this paper, we present some explicit exponents in the estimates for the volumes of sub-level sets of polynomials on bounded sets, and applications to the decay of oscillatory integrals and the convergent exponents of singular integrals. The methods used in this paper mainly come from geometry, which can be generalized to larger classes of sets and functions, such as definable sets in o-minimal structures (see Ta, 2021). But the case of polynomial functions have its own interests, where strict upper bounds and lower bounds are unknown for general polynomial functions. We obtain these bounds using geometric methods, and give some explicit exponents that depend only on the number of variables $n$, the degree $d$, and the order $k^{\prime}$ of $f$ at some point (see Theorem 3.2). Moreover, using these exponents from bounds for volumes of sub-level sets, we can determine the convergence of singular integrals of the form in Theorem 3.3.

More precisely, for $f: \mathbb{R}^{n} \rightarrow \mathbb{R}$, being a nonzero polynomial function of degree $d$, and $A$, a bounded domain in $\mathbb{R}^{n}$, we set $d^{\prime} \in \mathbb{N} \backslash\{0\}$ and $k^{\prime}=\operatorname{dim}\left(\left\{x \in f^{-1}(0): \operatorname{ord}_{x} f \geq\right.\right.$ $\left.\left.d^{\prime}\right\} \cap \operatorname{int} A\right)$. Then we prove in Theorem 2.5, Theorem 3.2, and Theorem 3.3 that there exist $C, C^{\prime}, C^{\prime \prime}>0$ such that

(1) $C^{\prime} t^{\alpha^{\prime}} \leq V(t) \leq C t^{\alpha}$ for all $t \geq 0$, where $\alpha=\frac{1}{d}$ and $\alpha^{\prime}=\frac{n-k^{\prime}}{d^{\prime}},\left(\alpha=\frac{n}{d}\right.$ for $f$ satisfying the condition given in Lemma 2.3),

(2) $I(\lambda) \leq C^{\prime \prime} \lambda^{-\beta}$ for all $\lambda>0$, where $\beta=\frac{1}{d}$, and

(3) $\int_{A}|f(x)|^{-\gamma} d x$ is convergent when $\gamma<\alpha$ and divergent when $\gamma \geq \alpha^{\prime}$. 
Now we explain the main ideas that lead to the above results.

To estimate the upper bound for the volume of a sub-level set of $f$ (see Theorem 2.5), we first choose a good direction for the zero-set, i.e., a linear subspace $V$ such that the projection $\left.p_{V}\right|_{f^{-1}(0)}: f^{-1}(0) \rightarrow V^{\perp}$ is a finite map. Then the restriction of the sublevel set on each fiber of $p_{V}$ has only isolated zeros. From these, we establish estimates for the volumes of the sub-level sets of polynomials in one variable with coefficients that depend on parameters to get the upper bounds for the volumes. To get the lower bounds, we use the homogeneous components to prove that the sub-level sets always contain balls with suitable radius.

For oscillatory integrals, we treat the behavior of the phase function $f$ nearby its critical points by applying Stationary Phase Principle. Similar to the proof in Ta (2021), Theorem 2, we partition the domain into two parts: on the first one $\|\nabla f\| \leq t$, and on the second one $\|\nabla f\|>t$. Applying the estimate for volumes of sub-level sets for the first part, and van der Corput's lemma for the second part, then scaling $t$ with $\lambda$, we get the estimate for the decay (see Theorem 3.2).

For singular integrals, since $V(t)=\operatorname{Vol}\left(K_{A, f, t}\right)$ is nondecreasing in $t$, by the Stieltjes integration we have $\int_{A}|f|^{-\gamma}=\int_{\mathbb{R}} t^{-\gamma} d V(t)$. Using integration by parts and the estimates for $V(t)$, we obtain the convergence information of the considered singular integrals (see Theorem 3.3).

The main theorems are Theorem 2.5, which is proved in Section 2, Theorems 3.2 and 3.3, which are proved in Section 3.

Notation: In this paper, we will denote the Euclidean norm in $\mathbb{R}^{n}$ by $\|\cdot\|$, the $k$-dimensional ball with center $a$ and radius $r$ by $B^{k}(a, r)$, the $n$-dimensional Lebesgue measure of $X \subset \mathbb{R}^{n}$ by $\operatorname{Vol}_{n}(X)$ or $\operatorname{Vol}(X)$, the gradient of $f$ by $\nabla f$, and the linear subspace generated by $v_{1}, \cdots, v_{k} \in \mathbb{R}^{n}$ by $L\left(v_{1}, \cdots, v_{k}\right)$.

\section{UPPER AND LOWER BOUNDS FOR THE VOLUMES}

In this section, we present some explicit exponents in the upper or lower estimates for the volumes of sub-level sets of polynomials on a bounded subset of $\mathbb{R}^{n}$. First, for polynomials of one variable, we have the following well-known estimates.

Lemma 2.1. Let $P(x)=a_{d} x^{d}+\cdots+a_{0}$ be a real polynomial and I be a bounded interval. Let $\lambda>0$. Then

(i) If there exists $p \geq 1$ such that $\left|P^{(p)}(x)\right| \geq \lambda$, for all $x \in I$, then

$$
\operatorname{Vol}_{1}\left(K_{I, P, t}\right) \leq C_{p}\left(\frac{t}{\lambda}\right)^{\frac{1}{p}}
$$

where $C_{p}$ is a constant depending only on $p$. 
(ii) If $\left|a_{0}\right|-\sup _{x \in I}\left|P(x)-a_{0}\right| \geq \lambda$, then

$$
\operatorname{Vol}_{1}\left(K_{I, P, t}\right) \leq C_{0}\left(\frac{t}{\lambda}\right)^{\frac{1}{d}}
$$

where $C_{0}=\operatorname{Vol}_{1}(I)$.

Proof.

For the proof of (i) see, for example, Carbery et al. (1999), Proposition 2.1. To prove (ii), note that for $0 \leq t<\lambda$, we have $|P(x)| \geq\left|a_{0}\right|-\left|P(x)-a_{0}\right| \geq \lambda>t$, and hence $\operatorname{Vol}_{1}\left(K_{I, P, t}\right)=0$. Moreover, for all $t$, we have $\operatorname{Vol}_{1}\left(K_{I, P, t}\right) \leq \operatorname{Vol}_{1}(I)$. Therefore, $\operatorname{Vol}_{1}\left(K_{I, P, t}\right) \leq \frac{\operatorname{Vol}(I)}{\lambda^{\frac{1}{d}}} t^{\frac{1}{d}}$

To apply the Lemma 2.1 to estimate volumes of sub-level sets of polynomials of $n$ variables, we need the following lemma.

Lemma 2.2. Let $g(a, s)=a_{0}+a_{1} s+\cdots+a_{d} s^{d}$ be a polynomial of degree $d$, where $a=$ $\left(a_{0}, \cdots, a_{d}\right)$. For $m, R>0$, set

$$
K=\left\{a=\left(a_{0}, \cdots, a_{d}\right) \in \mathbb{R}^{d+1}:\left|a_{0}\right|+\cdots+\left|a_{d}\right| \geq m\right\} \text { and } I=[-R, R] .
$$

Then there exists $\lambda=\lambda(d, m, R)>0$ such that for each $a \in K$, either there exists $p \in$ $\{1, \cdots, d\}$ such that

$$
\left|\frac{\partial^{p} g}{\partial s^{p}}(a, s)\right| \geq \lambda, \text { for all } s \in I, \text { or }\left|a_{0}\right|-\sup _{s \in I}\left|g(a, s)-a_{0}\right| \geq \lambda
$$

Proof.

First, we choose a family of positive numbers $\varepsilon_{d}, \cdots, \varepsilon_{0}$ satisfying the following conditions:

(1) $\varepsilon_{p}<\frac{m}{d+1}$, for all $p \in\{0, \cdots, d\}$.

(2) $p ! \varepsilon_{p}>(p+1) ! \varepsilon_{p+1} R+\cdots+\frac{d !}{(d-p) !} \varepsilon_{d} R^{d-p}$, for all $p \in\{d-1, \cdots, 0\}$.

For each $\varepsilon>0$, set

$$
A_{p}(\varepsilon)=\left\{a=\left(a_{0}, \cdots, a_{d}\right) \in K:\left|a_{p}\right| \leq \varepsilon\right\}, p \in\{d, \cdots, 0\} .
$$

For each $a \in K$, two cases are to be considered:

Case 1.d: $a \notin A_{d}\left(\varepsilon_{d}\right)$. Then $\left|\frac{\partial^{d} g}{\partial s^{d}}(a, s)\right|=\left|d ! a_{d}\right| \geq d ! \varepsilon_{d}=\lambda_{d}$, for all $s \in I$.

Case 2.d: $a \in A_{d}\left(\varepsilon_{d}\right)$. In this case there are two subcases to consider:

$$
a \in A_{d}\left(\varepsilon_{d}\right) \backslash A_{d-1}\left(\varepsilon_{d-1}\right) \text { or } a \in A_{d}\left(\varepsilon_{d}\right) \cap A_{d-1}\left(\varepsilon_{d-1}\right) .
$$

In general, the two cases to consider are: 
Case 1.p: $a \in A_{d}\left(\varepsilon_{d}\right) \cap \cdots \cap A_{p+1}\left(\varepsilon_{p+1}\right) \backslash A_{p}\left(\varepsilon_{p}\right)$.

Case 2.p: $a \in A_{d}\left(\varepsilon_{d}\right) \cap \cdots \cap A_{p+1}\left(\varepsilon_{p+1}\right) \cap A_{p}\left(\varepsilon_{p}\right)$.

However, by condition (1), there exists $q \in\{d, \cdots, 0\}$ such that $A_{d}\left(\varepsilon_{d}\right) \cap A_{d-1}\left(\varepsilon_{d-1}\right)$ $\cap \cdots \cap A_{q}\left(\varepsilon_{q}\right)=\emptyset$. Therefore, there exists $p=p(a) \in\{d, \cdots, 0\}$ such that $a$ falls in Case 1.p. In this case, by condition (2), we have

$$
\begin{aligned}
\left|\frac{\partial^{p} g}{\partial s^{p}}(a, s)\right| & =\left|p ! a_{p}+(p+1) ! a_{p+1} s+\cdots+\frac{d !}{(d-p) !} a_{d} s^{d-p}\right| \\
& \geq p !\left|a_{p}\right|-\left|(p+1) ! a_{p+1} s+\cdots+\frac{d !}{(d-p) !} a_{d} s^{d-p}\right| \\
& \geq p ! \varepsilon_{p}-\left[(p+1) ! \varepsilon_{p+1} R+\cdots+\frac{d !}{(d-p) !} \varepsilon_{d} R^{d-p}\right]=\lambda_{p}>0, \text { for all } s \in I .
\end{aligned}
$$

Let $\lambda=\min \left\{\lambda_{d}, \cdots, \lambda_{0}\right\}$. Then, by construction, it is easy to check that $\lambda$ satisfies the demand of the lemma.

Lemma 2.3. Let $f: \mathbb{R}^{n} \rightarrow \mathbb{R}$ be a polynomial of degree $d$. Assume that $f$ has an isolated zero at 0 and there is a constant $\varepsilon_{0}>0$ such that for all $x \in B\left(0, \varepsilon_{0}\right) \backslash\{0\}$

$$
\langle\nabla f(x), x\rangle \neq 0
$$

Then for all $u \in[0,1], x \in B\left(0, \varepsilon_{0}\right)$, we have

$$
|f(u x)| \leq|f(x)| \text {. }
$$
all $u \geq 0$.

In particular, sub-level set $K_{B\left(0, \varepsilon_{0}\right), f, t}$ is star-shaped with respect to center 0 , for

\section{Proof.}

Fix a point $x \in B\left(0, \varepsilon_{0}\right)$ and set $g(u)=f(u x), u \in[0, \infty]$. Since $\langle\nabla f(y), y\rangle \neq 0$ with $\left.y \in B\left(0, \varepsilon_{0}\right) \backslash\{0\}\right)$, we have

$$
g^{\prime}(u)=\langle\nabla f(u x), u x\rangle \neq 0, \forall u \in(0,1] .
$$

Then $g^{\prime}$ has a constant sign on $(0,1]$, and hence, $g$ is monotone on $[0,1]$. This implies

$$
\left|f\left(x^{\prime}\right)\right|=|g(u)| \leq|g(1)|=|f(x)|, \text { for all } x^{\prime}=u x \text { with } u \in[0,1] .
$$

The first part of the lemma is proved.

For the second part of the lemma, let $x \in K_{B\left(0, \varepsilon_{0}\right), f, t}$, and set $[0, x]=\left\{x^{\prime}: x^{\prime}=\right.$ $u x, u \in[0,1]\}$. Applying (1), we get

$$
\left|f\left(x^{\prime}\right)\right| \leq|f(x)| \leq t, \text { for all } x^{\prime} \in[0, x] .
$$

This implies that $x^{\prime} \in K_{B\left(0, \varepsilon_{0}\right), f, t}$, and hence, $[0, x] \subset K_{B\left(0, \varepsilon_{0}\right), f, t}$. This holds for all $x$ in $K_{B\left(0, \varepsilon_{0}\right), f, t}$, so the sub-level set $K_{B\left(0, \varepsilon_{0}\right), f, t}$ is star-shaped. 
Example 2.4. We give here a class of polynomial functions that satisfies the condition given in Lemma 2.3. Let $f: \mathbb{R}^{n} \rightarrow \mathbb{R}$ be a homogeneous polynomial of degree $d$ that has an isolated zero at 0 . By the homogeneous Euler identity, we have

$$
\langle\nabla f(x), x\rangle=d \cdot f(x) \neq 0, \forall x \neq 0,
$$

which satisfies the condition in Lemma 2.3. Therefore one can conclude that the sub-level set $\{x \in B(0, R):|f(x)| \leq u\}$ is star-shaped for any $R, u \geq 0$.

Now we come to the main theorem of this part.

Theorem 2.5. Let $f: \mathbb{R}^{n} \rightarrow \mathbb{R}$ be a nonzero polynomial function of degree $d$ and $A$ be a bounded subset of $\mathbb{R}^{n}$. Set

$$
V(t)=\operatorname{Vol}\left(K_{A, f, t}\right) \text {, and } Z_{d^{\prime}}(f)=\left\{x \in f^{-1}(0): \operatorname{ord}_{x} f=d^{\prime}\right\}\left(d^{\prime} \in \mathbb{N}\right) .
$$

Then the following assertions hold true:

(a) There exists $C=C(f, A)>0$ such that

$$
V(t) \leq C t^{\frac{1}{d}}, \text { for all } t \geq 0 .
$$

(b) If $\operatorname{dim}\left\{Z_{d^{\prime}}(f) \cap \operatorname{int} A\right\} \geq k^{\prime}$, then there exists $C^{\prime}=C^{\prime}\left(f, A, d^{\prime}, k^{\prime}\right)>0$ such that

$$
V(t) \geq C^{\prime} t^{\frac{n-k^{\prime}}{d^{\prime}}}, \text { for all } t \geq 0 .
$$

(c) If $0 \in \operatorname{int}(A) \neq \emptyset$ and $f$ satisfies the condition given in Lemma 2.3, then there exists $C^{\prime \prime \prime}(f, A)>0$ such that

$$
V(t) \leq C^{\prime \prime \prime} t^{\frac{n}{d}}, \text { for all } t \geq 0 .
$$

Proof.

(a) First, note that $V(t) \leq \operatorname{Vol}(\{x \in \bar{A}:|f(x)| \leq t\})$, we can assume that $A=\bar{A}$, i.e. $A$ is compact.

If $f^{-1}(0) \cap A=\emptyset$, then by compactness $V(t)=0$ for all $t \geq 0$ sufficiently small, and the desired inequality follows.

Now, suppose that $f^{-1}(0) \cap A \neq \emptyset$, and hence $\operatorname{dim} f^{-1}(0) \geq 0$. Let $R=\sup \{\|x\|$ : $x \in A\}$. One can observe that $f^{-1}(0)$ is an algebraic set of dimension $\leq n-1$, so by Parusiński (1994), Lemma 5.6, there exists a unit vector $e \in \mathbb{R}^{n}$ such that the following condition is satisfied:

$$
\operatorname{dim} V_{x} \cap f^{-1}(0) \text { for all } x \in \mathbb{R}^{n}, \text { where } V_{x}=x+\mathbb{R} e .
$$

We set $x=\hat{x}_{e}+x_{e} e$, where $x_{e}=\langle x, e\rangle$, and $f(x)=f_{e}\left(\hat{x}_{e}, x_{e}\right)=a_{0}\left(\hat{x}_{e}\right)+\cdots+a_{d}\left(\hat{x}_{e}\right) x_{e}^{d}$. Applying (2), one can imply that $\left.f\right|_{V_{x}}$ has only isolated zeros for all $x \in \mathbb{R}^{n}$. Thus, it follows that $a_{0}, \cdots, a_{d}$ have no common zero. Also, by the continuity of the $a_{j}$ term and the compactness of $A$, there exists $m>0$, such that

$$
m \leq\left|a_{0}\left(\hat{x}_{e}\right)\right|+\cdots+\left|a_{d}\left(\hat{x}_{e}\right)\right|, \text { for all } x \in A .
$$


Now the conditions in Lemma 2.2 are satisfied, so there exists $\lambda>0$ such that for each $x \in A$ either there exists $p \in\{1, \cdots, d\}$ such that

$$
\left|\frac{\partial^{p} f_{e}}{\partial x_{e}^{p}}\left(\hat{x}_{e}, x_{e}\right)\right| \geq \lambda, \text { for all } x_{e} \in I
$$

or $\left|a_{0}\left(\hat{x}_{e}\right)\right|-\sup _{x_{e} \in I}\left|f_{e}\left(\hat{x}_{e}, x_{e}\right)-a_{0}\left(\hat{x}_{e}\right)\right| \geq \lambda$.

Combined with parts (a) and (b) of Lemma 2.1, we get $C^{\prime}>0$, such that

$$
\operatorname{Vol}_{1}\left(\left\{x_{e} \in I:\left|f_{e}\left(\hat{x}_{e}, x_{e}\right)\right| \leq t\right\}\right) \leq C^{\prime} t^{\frac{1}{d}} \text {, for all } 0 \leq t \leq 1, x \in A .
$$

Next, applying Fubini's theorem to estimate the volume of the sub-level set, we have

$$
\operatorname{Vol}\left(K_{A, f, t}\right)=\operatorname{Vol}(\{x \in A:|f(x)| \leq t\}) \leq C^{\prime} t^{\frac{1}{d}} \operatorname{Vol}_{n-1}\left(p_{e}(A)\right) \leq C^{\prime \prime} t^{\frac{1}{d}} \text { for all } 0 \leq t \leq 1,
$$

where $p_{e}: \mathbb{R}^{n} \rightarrow e^{\perp}$ is the orthogonal projection along $e$, and $C^{\prime \prime}=C^{\prime} \operatorname{Vol}_{n-1}\left(p_{e}(A)\right)$.

Finally, taking $C=\max \left\{C^{\prime \prime}, \operatorname{Vol}(A)\right\}$, we get

$$
V(t)=\operatorname{Vol}\left(K_{A, f, t}\right) \leq C t^{\frac{1}{d}} \text {, for all } t \geq 0 .
$$

(b) Since $Z_{d^{\prime}}(f)$ is a semialgebraic set and, by supposition, $\operatorname{dim} Z_{d^{\prime}}(f) \cap \operatorname{int} A \geq k^{\prime}$, there exists a manifold $\Gamma \subset Z_{d^{\prime}}(f) \cap \operatorname{int} A$ with $\operatorname{dim} \Gamma=k^{\prime}$. Moreover, $\Gamma$ can be chosen so that there exists an $\left(n-k^{\prime}\right)$-dimensional linear subspace $V$ of $\mathbb{R}^{n}$ such that the restriction to $\Gamma$ of the orthogonal projection along $V,\left.p_{V}\right|_{\Gamma}: \Gamma \rightarrow p_{V}(\Gamma) \subset V^{\perp}$, is a bi-Lipschitz homeomorphism.

Let $\left(e_{1}, \cdots, e_{n}\right)$ be an orthogonal basis of $\mathbb{R}^{n}$ such that $V=L\left(e_{1}, \cdots, e_{n-k^{\prime}}\right)$. For this basis, we write

$$
x=\left(x_{1}, \cdots, x_{n}\right), \bar{x}=\left(x_{1}, \cdots, x_{n-k^{\prime}}\right) \in V, \hat{x}=\left(x_{n-k^{\prime}+1}, \cdots, x_{n}\right) \in V^{\perp} .
$$

For points $a \in \Gamma$ and $x=(\bar{a}+\bar{u}, \hat{a}) \in V_{a}=a+V$, we set

$$
\left.f\right|_{V_{a}}(x)=g_{\hat{a}}(\bar{u})=g_{\hat{a}, d^{\prime}}(\bar{u})+\cdots+g_{\hat{a}, d}(\bar{u}),
$$

where $g_{\hat{a}, j}$ is the homogeneous component of degree $j$ of $g_{\hat{a}}$. Then for all $x=(\bar{a}+\bar{u}, \hat{a}) \in$ $V_{a}=a+V$ with $0<\|\bar{u}\|<1$,

$$
\begin{aligned}
|f(x)|=\left|g_{\hat{a}}(\bar{u})\right| & \leq\left|g_{\hat{a}, d^{\prime}}(\bar{u})\right|+\cdots+\left|g_{\hat{a}, d}(\bar{u})\right| \\
& \leq\left|g_{\hat{a}, d^{\prime}}\left(\frac{\bar{u}}{\|\bar{u}\|}\right)\right|\|\bar{u}\|^{d^{\prime}}+\cdots+\left|g_{\hat{a}, d}\left(\frac{\bar{u}}{\|\bar{u}\|}\right)\right|\|\bar{u}\|^{d} \\
& \leq \max \left\{\left|g_{\hat{a}, d^{\prime}}(\bar{v})\right|+\cdots+\left|g_{\hat{a}, d}(\bar{v})\right|:\|\bar{v}\|=1\right\}\|\bar{u}\|^{d^{\prime}} .
\end{aligned}
$$

By the continuity of the $g_{\hat{a}, j}$ term, and by shrinking $\Gamma$ if necessary, we have 


$$
M=\sup \left\{\left|g_{\hat{a}, d^{\prime}}(\bar{v})\right|+\cdots+\left|g_{\hat{a}, d}(\bar{v})\right|: a \in \Gamma,\|\bar{v}\|=1\right\}<+\infty .
$$

From the above estimate, for all $a \in \Gamma$ and $x=(\bar{a}+\bar{u}, \hat{a}) \in V_{a}$ with $\|\bar{u}\|<M^{-\frac{1}{d^{\prime}} t} \frac{1}{d^{\prime}}<$ 1, we have $|f(x)|<t$, i.e. $K_{A, f, t} \cap V_{a}$ contains an $\left(n-k^{\prime}\right)$-dimensional ball of radius $M^{-\frac{1}{d^{\prime}}} t \frac{1}{d^{\prime}}$. By the Fubini theorem or the coarea formula (see Federer, 1969), we get the lower bound for the volume of a sub-level set

$$
V(t) \geq \operatorname{Vol}_{k^{\prime}}\left(p_{V}(\Gamma)\right) \operatorname{Vol}_{n-k^{\prime}}\left(B^{n-k^{\prime}}\left(0, M^{-\frac{1}{d^{\prime}}} t^{\frac{1}{d^{\prime}}}\right)\right) \geq \operatorname{Vol}_{k^{\prime}}\left(p_{V}(\Gamma)\right) M^{-\frac{n-k^{\prime}}{d^{\prime}}} t^{\frac{n-k^{\prime}}{d^{\prime}}},
$$

for all $t>0$ sufficiently small. From this (ii) follows.

(c) Let $\left\{e_{1}, \ldots, e_{n}\right\}$ be a basis of $\mathbb{R}^{n}$. Since $f$ has an isolated zero $0 \in A$, from Lemma 2.3, the condition

$$
\text { (*) } \operatorname{dim}\left(V_{x} \cap f^{-1}(0)\right) \leq 0
$$

holds for all $x \in A$ and $V_{x}=x+\mathbb{R} e$, where $e \in \mathbb{R}^{n} \backslash\{0\}$ is any direction. Then one can follow the proof in (i). For each $e \in S^{n-1}$, there exists $C_{e}^{\prime}>0$, such that

$$
\operatorname{Vol}_{1}\left(\left\{x_{e} \in I:\left|f_{e}\left(\hat{x}_{e}, x_{e}\right)\right| \leq t\right\}\right) \leq C_{e}^{\prime} t^{1 / d} \text { for all } 0 \leq t \leq 1, x \in A .
$$

By Lemma 2.3, there exists $\varepsilon_{0}>0$ such that the sub-level set

$$
K_{A \cap B\left(0, \varepsilon_{0}\right), f, t}=\left\{x \in A \cap B\left(0, \varepsilon_{0}\right):|f(x)| \leq t\right\}
$$

is bounded and star-shaped. By using a suitable change of coordinates, we can choose the direction $e_{1}$ in the basis of $\mathbb{R}^{n}$ so that $C_{e_{1}}^{\prime} \geq C_{e}$ for all $e \in \mathbb{R}^{n}$.

If we put $t_{0}=\min \left\{1,\left(\frac{\varepsilon_{0}}{C_{e_{1}}^{\prime}}\right)^{d}\right\}$, then

$$
K_{A \cap B\left(0, \varepsilon_{0}\right), f, t} \subset B\left(0, C_{e_{1}}^{\prime} t^{1 / d}\right) \subset B\left(0, \varepsilon_{0}\right), \text { for all } t \in\left[0, t_{0}\right] .
$$

Therefore, for all $t \in\left(0, t_{0}\right]$, we have

$$
\operatorname{Vol}\left(K_{A \cap B\left(0, \varepsilon_{0}\right), f, t}\right) \leq \operatorname{Vol}\left(B\left(0, C_{e_{1}}^{\prime} t^{1 / d}\right)\right) \leq \operatorname{Vol}(B(0,1))\left(C_{e_{1}}^{\prime}\right)^{n} t^{n / d} .
$$

Finally, by the compactness of $A$, we see that $V(t) \leq \operatorname{Vol}(A) \leq \frac{\operatorname{Vol}(A)}{t_{0}^{n / d}}$ when $t_{0} \leq t$. So we can choose $C^{\prime \prime \prime}=\max \left\{\operatorname{Vol}(B(0,1))\left(C_{e_{1}}^{\prime}\right)^{n}, \frac{\operatorname{Vol}(A)}{t_{0}^{n / d}}\right\}$ to get the upper bound

$$
V(t)=\operatorname{Vol}(\{x \in A:|f(x)| \leq t\}) \leq C^{\prime \prime \prime} t^{\frac{n}{d}} \text {, for all } t \geq 0 .
$$

Corollary 2.6. Let $f: \mathbb{R}^{n} \rightarrow \mathbb{R}$ be a nonzero polynomial function of degree $d$ and $A$ be a bounded subset of $\mathbb{R}^{n}$. Then 
(a) If $f^{-1}(0) \cap \operatorname{int} A \neq \emptyset$, then there exists $C, C^{\prime}>0$ such that

$$
C^{\prime} t^{n} \leq V(t) \leq C t^{\frac{1}{d}}, \text { for all } t \geq 0 \text {. }
$$

(b) If there exists $a \in f^{-1}(0) \cap \operatorname{int} A$ with $\operatorname{ord}_{a} f=d^{\prime}$, then there exists $C^{\prime \prime}>0$ such that

$$
V(t) \geq C^{\prime \prime} t^{\frac{n}{d^{t}}}, \text { for all } t \geq 0 \text {. }
$$

Proof.

Apply Theorem 2.5 with $d^{\prime}=1$ for (a), and $k^{\prime}=0$ for (b).

In the sequel, we will denote $f(t) \precsim g(t)$ if and only if there exists $C>0$ so that $f(t) \leq C g(t)$, for all $t>0$ sufficiently small, and $f(t) \approx g(t)$ if and only if $g(t) \precsim f(t)$ and $f(t) \precsim g(t)$.

\section{Remark 2.1.}

(i) The exponents in Theorem 2.5 are explicit and easy to calculate (see Carbery et al., 1999, Theorem 7.1; Dieu et al., 2018, Theorem 3.1, 3.2).

(ii) Due to the lower-bounds, in some cases, we can obtain the first exponent in the asymptotic expansion of $V(t)$, i.e. the exponent $\alpha_{f}$ so that $V(t) \approx t^{\alpha_{f}}$ (see the examples below). From Theorem 2.5, we have $\alpha_{f} \in\left[\frac{1}{d}, \frac{n-k^{\prime}}{d^{\prime}}\right]$. In the case where $f$ satisfies the condition in Lemma 2.3, we get $\alpha_{f} \in\left[\frac{n}{d}, \frac{n-k^{\prime}}{d^{\prime}}\right]$. Nevertheless, in general, the data $(n, d=$ $\left.\operatorname{deg} f, k=\operatorname{dim} f^{-1}(0), d^{\prime}=\operatorname{ord}_{a} f, k^{\prime}=\operatorname{dim} Z_{d^{\prime}}(f)\right)$ are not sufficient to get $\alpha_{f}$. For the case where $f$ is a homogeneous polynomial of even degree $d$ under the assumption that the sub-level set has a finite volume on $\mathbb{R}^{n}$, it is proved that $\alpha_{f}=\frac{n}{d}$ (see Lasserre, 2015, Theorem 2.2; Morozov \& Shakirov, 2009; Morozov \& Shakirov, 2010).

\section{Example 2.7.}

a) Let $f$ be a polynomial of degree $d$ in $n$ variables and $A$ be a bounded set. If $f$ has $\operatorname{dim} f^{-1}(0)=n-1$ and there exists $a \in f^{-1}(0)$ such that $\operatorname{ord}_{a} f=d$, then $V(t) \approx t^{\frac{1}{d}}$.

b) For $f$ be a homogeneous polynomial of degree $d$ in $n$ variables, we can not in general have $V(t) \approx t^{\frac{n-k}{d}}, \forall t \geq 0, k=\operatorname{dim} f^{-1}(0)$.

For example, consider $f(x, y)=x^{2} y^{2}\left(x^{2}+y^{2}\right)$ on $A=[0,1]^{2}$. We have $f^{-1}(0)=$ $O x \cup O y$ and $\operatorname{dim} f^{-1}(0)=1$; hence, by Theorem 2.5 , we get $C_{1}, C_{2}>0$ :

$$
C_{1} t^{\frac{1}{2}} \leq V(t) \leq C_{2} t^{\frac{1}{6}}, \forall t \geq 0
$$

By Lasserre (2015), Theorem 2.2, we have $V(t) \approx t^{\frac{1}{3}}$.

\section{APPLICATIONS}

In this section, we apply Theorem 2.5 to give some explicit exponents in the estimates of the decay of oscillatory integrals with polynomial phase functions, and the 
convergence of integrals of the form $\int_{A}|f|^{-\gamma}$, where $f$ is a polynomial and $A$ is a bounded domain in $\mathbb{R}^{n}$.

First, we recall the van der Corput lemma.

Lemma 3.1. (van der Corput) Let $f:(a, b) \rightarrow \mathbb{R}$ be a $C^{1}$ function. Fix $t>0$. Suppose that $\left|f^{\prime}(x)\right| \geq t, \forall x \in(a, b)$, and $f^{\prime}$ is monotonic. Then

$$
\left|\int_{a}^{b} e^{i \lambda f(x)} d x\right| \leq 3(\lambda t)^{-1}, \text { for all } \lambda>0
$$

Proof.

See, for example, Stein (1993), Chapter VIII, Proposition 2, or Carbery et al. (1999), Proposition 2.2.

For $g: \mathbb{R}^{n} \rightarrow \mathbb{R}$ being a $C^{1}$ function with compact support, set

$$
\|g\|_{\infty}=\sup _{\mathbb{R}^{n}}|g|, \text { and }\|\nabla g\|_{1}=\int_{\mathbb{R}^{n}}\|\nabla g\|
$$

Theorem 3.2. Let $f: \mathbb{R}^{n} \rightarrow \mathbb{R}$ be a polynomial function of degree $d \geq 1$. Then for any semialgebraic compact subset $A$ of $\mathbb{R}^{n}$, there exists $C=C(f, A)>0$ such that for any $C^{1}$ function $g: \mathbb{R}^{n} \rightarrow \mathbb{R}$ with compact support containing in $A$, we have

$$
\left|\int_{A} e^{i \lambda f(x)} g(x) d x\right| \leq C \lambda^{-\frac{1}{d}}\left(\|g\|_{\infty}+\|\nabla g\|_{1}\right), \text { for all } \lambda>0 \text {. }
$$

Proof. (see Loi, 2021)

For each $t>0$, put $A=A_{t} \cup B_{t}$, where

$$
A_{t}=\{x \in A:\|\nabla f(x)\| \leq t\}, B_{t}=\{x \in A:\|\nabla f(x)\|>t\} .
$$

We will estimate the integrals on each set of the union.

To estimate the integral on $A_{t}$, we apply Theorem 2.5 to get $C_{1}>0$ so that

$$
\operatorname{Vol}\left(A_{t}\right)=\operatorname{Vol}\left(\left\{x \in A:\|\nabla f(x)\|^{2} \leq t^{2}\right\}\right) \leq C_{1} t^{\frac{1}{d-1}} .
$$

Therefore,

$$
\left|\int_{A_{t}} e^{i \lambda f(x)} g(x) d x\right| \leq \operatorname{Vol}\left(A_{t}\right)\|g\|_{\infty} \leq C_{1} t^{\frac{1}{d-1}}\|g\|_{\infty} .
$$

To estimate the integral on $B_{t}$, we apply the van der Corput lemma (Lemma 3.1). First, note that

$$
B_{t} \subset \bigcup_{k=1}^{n}\left\{x \in A:\left|\partial_{k} f(x)\right| \geq \frac{t}{n}\right\},
$$

For $k=n$, let $x=\left(x^{\prime}, x_{n}\right)$ denote a point in $B_{t} \subset \mathbb{R}^{n-1} \times I, \tilde{B}_{t}$ denote the projection of $B_{t}$ to the first $n-1$ coordinates, and $I$ denote an interval so that $B_{t} \subset \tilde{B}_{t} \times I$. For each 
$x^{\prime} \in \tilde{B}_{t}$, since $B_{t}$ is a semialgebraic set, there exists $N \in \mathbb{N}$, independent of $x^{\prime}$ and $t$, such that the set

$$
\left\{x_{n} \in I:\left|\partial_{n} f\left(x^{\prime}, x_{n}\right)\right| \geq \frac{t}{n}\right\}
$$

is the union of atmost $d$ intervals, say $I_{1}, \cdots, I_{d}$ (The intervals depend on $x^{\prime}$ and $t$, and some may be empty). On each of the intervals, say $I_{j}=(a, b)$, let $F\left(x^{\prime}, x_{n}\right)=\int_{a}^{x_{n}} e^{i \lambda f\left(x^{\prime}, s\right)} d s, x_{n} \in$ $I_{j}$. Since $\left|\partial_{n} f\left(x^{\prime}, x_{n}\right)\right| \geq t / n$ on $I_{j}$, by the van der Corput lemma (Lemma 3.1), $\left|F\left(x^{\prime}, x_{n}\right)\right| \leq$ $3\left(\frac{\lambda t}{n}\right)^{-1}$. Integrating by parts and using this inequality, we get

$$
\begin{aligned}
\left|\int_{I_{j}} e^{i \lambda f\left(x^{\prime}, x_{n}\right)} g\left(x^{\prime}, x_{n}\right) d x_{n}\right| & =\left|\int_{I_{j}} \partial_{n} F\left(x^{\prime}, x_{n}\right) g\left(x^{\prime}, x_{n}\right) d x_{n}\right| \\
& \leq 3\left(\frac{\lambda t}{n}\right)^{-1} 2\|g\|_{\infty}+3\left(\frac{\lambda t}{n}\right)^{-1} \int_{I_{j}}\left|\partial_{n} g\left(x^{\prime}, x_{n}\right)\right| d x_{n} .
\end{aligned}
$$

Applying the Fubini theorem and the above estimation on each of the intervals, we get

$$
\begin{aligned}
\left|\int_{\left|\partial_{n} f\right| \geq \frac{t}{n}} e^{i \lambda f(x)} g(x) \chi_{B_{t}}(x) d x\right| & \leq \int_{\tilde{B}_{t}}\left(\sum_{j=1}^{d}\left|\int_{I_{j}} e^{i \lambda f\left(x^{\prime}, x_{n}\right)} g\left(x^{\prime}, x_{n}\right) d x_{n}\right|\right) d x^{\prime} \\
& \leq \int_{\tilde{B}_{t}}\left(\sum_{j=1}^{d}\left(3\left(\frac{\lambda t}{n}\right)^{-1}\left(2\|g\|_{\infty}+\int_{I_{j}}\left|\partial_{n} g\left(x^{\prime}, x_{n}\right)\right| d x_{n}\right)\right)\right) d x^{\prime} \\
& \leq C_{2}(\lambda t)^{-1}\left(\|g\|_{\infty}+\|\nabla g\|_{1}\right),
\end{aligned}
$$

where $C_{2}=\max _{1 \leq k \leq n} \operatorname{Vol}_{n-1}\left(p_{k}(A)\right) d 6 n$, and $p_{k}: \mathbb{R}^{n} \rightarrow \mathbb{R}^{n-1}$ is the projection missing the $k$-th coordinate.

Using similar estimations for $k=1,2, \cdots$, we get $C_{3}>0$ so that

$$
\begin{aligned}
\left|\int_{B_{t}} e^{i \lambda f(x)} g(x) d x\right| & \leq \sum_{k=1}^{n}\left|\int_{\left|\partial_{k} f\right| \geq \frac{t}{n}} e^{i \lambda f(x)} g(x) \chi_{B_{t}}(x) d x\right| \\
& \leq C_{3}(\lambda t)^{-1}\left(\|g\|_{\infty}+\|\nabla g\|_{1}\right) .
\end{aligned}
$$

From the above estimates, we have

$$
\begin{aligned}
\left|\int_{A} e^{i \lambda f(x)} g(x) d x\right| & \leq\left|\int_{A_{t}} e^{i \lambda f(x)} g(x) d x\right|+\left|\int_{B_{t}} e^{i \lambda f(x)} g(x) d x\right| \\
& \leq\left(C_{1} t^{\frac{1}{d-1}}+C_{3}(\lambda t)^{-1}\right)\left(\|g\|_{\infty}+\|\nabla g\|_{1}\right)
\end{aligned}
$$

Now we choose $t=\lambda^{-\delta}$, so that $t^{\frac{1}{d-1}}=(\lambda t)^{-1}$, to get $\delta=\frac{d-1}{d}$, and hence

$$
\left|\int_{A} e^{i \lambda f(x)} g(x) d x\right| \leq C \lambda^{-\frac{1}{a}}\left(\|g\|_{\infty}+\|\nabla g\|_{1}\right), \text { for all } \lambda>0
$$

where $C=C_{1}+C_{3}$.

Theorem 3.3. Let $f: \mathbb{R}^{n} \rightarrow \mathbb{R}$ be a nonzero polynomial function. Let $A$ be a bounded subset $\mathbb{R}^{n}$. Put $V(t)=\operatorname{Vol}(\{x \in A:|f(x)| \leq t\})$. 
(a) If $V(t) \leq C t^{\alpha}$ for all $t \geq 0$ sufficiently small, then $\int_{A}|f(x)|^{-\gamma} d x<+\infty$, when $\gamma<\alpha$.

(b) If $V(t) \geq C^{\prime} t^{\alpha^{\prime}}$ for all $t \geq 0$ sufficiently small, then $\int_{A}|f(x)|^{-\gamma} d x=\infty$, when $\gamma \geq \alpha^{\prime}$

As a consequence, when $\operatorname{deg} f=d$, and $\operatorname{dim} Z_{d^{\prime}}(f) \cap \operatorname{int} A \geq k^{\prime}$,one can choose $\alpha=\frac{1}{d}, \alpha^{\prime}=\frac{n-k^{\prime}}{d^{\prime}}$. If $f$ satisfies the condition given in Lemma 2.3 , then $\alpha=\frac{n}{d}$.

Proof.

Note that $V(t)=\operatorname{Vol}(\{x \in A:|f(x)| \leq t\})$ is a nondecreasing function in $t$. Let $T=\sup _{A}|f|$. By a change of variables, (see Federer, 1969)

$$
\int_{A}|f(x)|^{-\gamma} d x=\int_{0}^{T} t^{-\gamma} d V(t) .
$$

Applying integration by parts, (see Federer, 1969), we have

$$
\int_{0}^{T} t^{-\gamma} d V(t)=\left.t^{-\gamma} V(t)\right|_{0} ^{T}+\gamma \int_{0}^{T} t^{-\gamma-1} V(t) d t .
$$

(a) If $V(t) \leq C t^{\alpha}$, then $0 \leq t^{-\gamma} V(t) \leq C t^{-\gamma+\alpha}$ and $\int_{0}^{T} t^{-\gamma-1} V(t) d t \leq \int_{0}^{T} C t^{-\gamma-1+\alpha} d t$. Therefore, $\left.t^{-\gamma} V(t)\right|_{0} ^{T}<+\infty$ and $\gamma \int_{0}^{T} t^{-\gamma-1} V(t) d t<+\infty$ when $\gamma<\alpha$, and hence, $\int_{A}|f(x)|^{-\gamma} d x<+\infty$, when $\gamma<\alpha$.

(b) Similarly, if $V(t) \geq C^{\prime} t^{\alpha^{\prime}}$, then $t^{-\gamma} V(t) \geq C^{\prime} t^{-\gamma+\alpha^{\prime}}$ and $\int_{0}^{T} t^{-\gamma-1} V(t) d t \geq$ $\int_{0}^{T} C^{\prime} t^{-\gamma-1+\alpha^{\prime}} d t$. Therefore, $\left.t^{-\gamma} V(t)\right|_{0} ^{T}=+\infty$ and $\gamma \int_{0}^{T} t^{-\gamma-1} V(t) d t=+\infty$ when $\gamma \geq \alpha^{\prime}$, and hence, the considered integral is divergent when $\gamma \geq \alpha^{\prime}$. The last part of the theorem is followed from Theorem 2.5.

Remark 3.1. The integration index of $f$ on $A$, is defined by

$$
i(f, A)=\sup \left\{\gamma: \int_{A}|f(x)|^{-\gamma} d x<+\infty\right\} .
$$

By Theorem 2.5 and Theorem 3.3, $i(f, A) \in\left[\frac{1}{d}, \frac{n-k^{\prime}}{d^{\prime}}\right]$, for any polynomial function $f$. In particular, $i(f, A) \in\left[\frac{n}{d}, \frac{n-k^{\prime}}{d^{\prime}}\right]$, when $f$ satisfies the condition in Lemma 2.3. Therefore, when the endpoints are equal, $i(f, A)$ is determined. But there are cases where $i(f, A)>\frac{1}{d}$ See the following examples.

\section{Example 3.4.}

a) From Theorem 3.3 and Example 2.7, if $f$ be a polynomial of degree $d$ in $n$ variables and $A$ be a bounded set, $\operatorname{dim} f^{-1}(0)=n-1$ and there exists $a \in f^{-1}(0)$ such that $\operatorname{ord}_{a} f=d$, then in a bounded neighborhood $A$ of the origin we have $\int_{A}|f(x)|^{-\gamma} d x<+\infty$ if and only if $\gamma<i(f, A)=\frac{1}{d}$. 
b) Let $g(x, y)=x^{2}+y^{2}$, and $A=\left\{(x, y) \in \mathbb{R}^{2}: x^{2}+y^{2} \leq 1\right\}$. Then we have $d=$ $\operatorname{deg} g=2, f^{-1}(0)=\{(0,0)\}$ has dimension 0 . Then, by Theorem 2.5, (c), we have

$$
t=t^{\frac{n-k^{\prime}}{d^{\prime}}} \leq V(t) \leq t^{\frac{n}{d}}=t
$$

By the above remark, we have $i(g, A)=1$.

c) In the above Theorem 3.3, if $f^{-1}(0) \cap A=\emptyset$ or $\forall d^{\prime} \in \mathbb{N}, \operatorname{dim} Z_{d^{\prime}} \cap \operatorname{int} A=\emptyset$, then $i(f, A)$ can be equal $\infty$.

\section{ACKNOWLEDGMENTS}

This research is partially supported by the Vietnamese National Foundation for Science and Technology Development (NAFOSTED) - Project ID 101.04-2017.324.

\section{REFERENCES}

Arnold, V. I., Gusein-Zade, S. M., \& Varchenko, A. N. (1988). Singularities of differentiable maps: Vol. II. Monodromy and asymptotics of integrals (Monographs in mathematics, Vol. 83). Birkhäuser. https://doi.org/10.1007/978-1-4612-3940-6

Carbery, A., Christ, M., \& Wright, J. (1999). Multidimensional van der Corput and sublevel set estimates. Journal of the American Mathematical Society, 12(4), 981 -1015. https://doi.org/10.1090/S0894-0347-99-00309-4

Cluckers, R., \& Miller, D. J. (2016). Bounding the decay of oscillatory integrals with a constructible amplitude function and a globally subanalytic phase function. Journal of Fourier Analysis and Applications, 22(1), 215-236. https://doi.org/10.1007 /s00041-015-9421-2

Federer, H. (1969). Geometric measure theory. Springer-Verlag.

Lasserre, J. B. (2015). A generalization of Löwner-John's ellipsoid theorem. Mathematical Programming, Series A, 152(1-2), 559-591. https://doi.org/10.1007/s10107 $-014-0798-5$

Morozov, A. Y., \& Shakirov, Sh. R. (2009). Introduction to integral discriminants. Journal of High Energy Physics, 12, 1-35. https://iopscience.iop.org/article/10.1088/ 1126-6708/2009/12/002/pdf

Morozov, A. Y., \& Shakirov, Sh. R. (2010). New and old results in resultant theory. Theoretical and Mathematical Physics, 163, 587-617. https://doi.org/10.1007/s1123 2-010-0044-0

Nguyen, N. D., Dau, H. H., Pham, T. S., \& Hoang, T. A. (2018). Volume estimates of sublevel sets of real polynomials. Annales Polonici Mathematici, 121(2), 157 -174. https://doi.org/10.4064/ap170913-25-4

Parusiński, A. (1994). Lipschitz stratification of subanalytic sets. Annales scientifiques de l'É.N.S., série 4, 27(6), 661-696. https://doi.org/10.24033/asens.1703

Phong, D. H., Stein, E. M., \& Sturm, J. A. (1999). On the growth and stability of real-ana 
-lytic functions. American Journal of Mathematics, 121(3), 519-554. https://doi. org/10.1353/ajm.1999.0023

Rogers, K. M. (2005). Sharp van der Corput estimates and minimal divided differences. Proceedings of the American Mathematical Society, 133(12), 3543-3550. https: //doi.org/10.1090/S0002-9939-05-07918-9

Stein, E. M. (1993). Harmonic analysis: Real-variable methods, orthogonality and oscillatory integrals. Princeton University Press. https://doi.org/10.1515/9781400883 929

Ta, L. L. (2021). Volumes of sub-level sets and the decay of oscillatory integrals. Journal of Mathematical Inequalities, 15(2), 767-779. https://doi.org/10.7153/jmi2021-15-54 